Vol. 23 (2013) 1-10

(C) World Scientific Publishing Company

DOI: 10.1142/S2010194513010994

\title{
RECENT DEVELOPMENTS IN THE SEARCH FOR COMPTON-THICK AGN
}

\author{
I. GEORGANTOPOULOS \\ Osservatorio Astronomico di Bologna, INAF \\ Via Ranzani 1, Bologna, 40127, Italy \\ ioannis.georgantopoulos@oabo.inaf.it
}

\begin{abstract}
I present a review of X-ray and mid-IR surveys for Compton-thick Active Galactic Nuclei (AGN). These are the most highly obscured sources having hydrogen column densities $>1.5 \times 10^{24} \mathrm{~cm}^{-2}$. Key surveys in the local Universe are presented including the high energy SWIFT/BAT and INTEGRAL surveys, mid-IR and also optical surveys. Recently, deep X-ray surveys with Chandra and XMM-Newton have produced a number of candidate Compton-thick AGN at higher redshift primarily in the Chandra Deep Field South region. In addition, mid-IR surveys with Spitzer have helped to develop novel complementary techniques for the selection of Compton-thick AGN. The mid-IR techniques used to identify Compton-thick AGN include: a) $24 \mu \mathrm{m}$ excess sources relative to their optical emission b) Spitzer spectroscopy for the detection of high optical depth Si $9.7 \mu \mathrm{m}$ absorption features c) low X-ray to $6 \mu \mathrm{m}$ luminosity ratio.
\end{abstract}

Keywords: X-rays; Active Galactic Nuclei; Seyfert galaxies.

\section{Introduction}

The deep X-ray Universe has been probed at unparalleled depth thanks to the Chandra mission. The deepest Chandra X-ray surveys revealed an AGN sky density above $10.000 \mathrm{deg}^{-2}$ (Alexander et al. 2003, Luo et al. 2008, Xue et al. 2011), probing a flux limit of $f_{2-10 \mathrm{keV}} \approx 5 \times 10^{-17} \mathrm{erg} \mathrm{cm}^{-2} \mathrm{~s}^{-1}$ (for a review of X-ray surveys see Brandt \& Hasinger 2005). In contrast, the optical surveys for QSOs reach sky densities of about few hundred per square degree at a magnitude limit of $B=22$ mag (e.g. Wolf et al. 2003). Therefore, hard (2-10 keV) X-ray surveys have provided so far the most powerful method for the detection of AGN.

However, even the efficient hard X-ray surveys may be missing a substantial fraction of the most heavily obscured sources, the Compton-thick AGN, which have column densities $>10^{24} \mathrm{~cm}^{-2}$. At these high column densities, the attenuation of $\mathrm{X}$-rays is mainly due to Compton-scattering rather than photoelectric absorption. Although there are only a few dozen of Compton-thick AGN identified in the local Universe, (for a review see Comastri 2004), there is concrete evidence for the presence of a much higher number. The peak of the X-ray background at $20-30 \mathrm{keV}$ 
(Frontera et al. 2007, Churazov et al. 2007, Moretti et al. 2009) can be reproduced by invoking a significant number of Compton-thick sources at moderate redshifts. However, the exact density of Compton-thick sources required by X-ray background synthesis models still remains an open issue (Gilli et al. 2007, Sazonov et al. 2008, Treister et al. 2009, Ballantyne et al. 2011). Additional evidence for the presence of a numerous Compton-thick population comes from the directly measured space density of black holes in the local Universe (Soltan1982). It is found that this space density is a factor of 1.5-2 higher than that predicted from the X-ray luminosity function (Marconi et al. 2004, Merloni \& Heinz 2008), although the exact number depends on the assumed efficiency in the conversion of gravitational energy to radiation.

In this paper, we present a brief review of Compton-thick AGN. We start from X-ray spectroscopic results of nearby compton-thick AGN. We then discuss X-ray surveys for Compton-thick sources in both the local universe $(\mathrm{z}<0.1)$ as well as deep X-ray surveys which probe moderate redshifts where the bulk of the X-ray background is produced.

\section{X-Ray Spectroscopy of Compton-Thick AGN}

When the absorbing column exceeds $10^{24} \mathrm{~cm}^{-2}$, the attenuation in X-ray wavelengths is because of electron scattering rather than photoelectric absorption. The main characteristics of a Compton-thick spectrum are: a) a flat spectrum with $\Gamma \sim 1$ at energies below $10 \mathrm{keV}$. This originates in reflection on the back-side of the obscuring screen (George \& Fabian 1991, Matt et al. 2004, Murphy \& Yaqoob 2010). The flat spectrum is due to the combination Compton down-scattering of high energy photons to lower energies and subsequent photoelectric absorption. b) an $F e K \alpha$ line with large equivalent width, usually of the order of $1 \mathrm{keV}$ (e.g. Fukazawa et al. 2011); note however, that there are some exceptions of Compton-thick AGN with a small FeK $\alpha$ equivalent-width: e.g. Mrk-231 with an equivalent width of $\sim 300 \mathrm{eV}$ (Braito et al. 2004). c) An absorption turnover at energies above $10 \mathrm{keV}$, with the exact cut-off energy depending on the column density. NGC1068 represents an extreme case study of a Compton-thick AGN. This is one of the nearest AGN at a redshift of $z=0.038$. It has a column density of about $10^{25} \mathrm{~cm}^{-2}$ as derived from BeppoSAX observations (Matt et al. 2004). At such high column densities $\left(>5 \times 10^{24} \mathrm{~cm}^{-2}\right)$, the transmitted component is totally blocked by the obscuring material. The observed X-ray flux, arising from the back-side of the obscuring screen, is diminished by almost two orders of magnitude. NGC3079 is another example of a Compton-thick AGN. This is mildly Compton-thick having a column density of $\sim 2 \times 10^{24} \mathrm{~cm}^{-2}$. For a detailed discussion of various Compton-thick AGN broad-band SUZAKU spectra selected by SWIFT/BAT, see Comastri et al. (2010). An inventory of BeppoSAX observations of nearby AGN, including many Compton-thick AGN can be found in Dadina (2007). 


\section{Compton-Thick AGN in the Local Universe}

Comastri (2004) presented a sample from the literature of few tens of Comptonthick AGN in the local Universe. These are classified as Compton-thick AGN on the basis of BeppoSAX observations. Here, we present an update of this review citing the most recent results on the fraction of Compton-thick AGN among nearby AGN. All the results presented come from direct X-ray spectroscopy, either from SWIFT/BAT or from XMM-Newton.

\subsection{Ultra-hard (>10 keV) X-ray selected $A G N$}

The INTEGRAL and SWIFT missions explored the X-ray sky at high energies (15$200 \mathrm{keV}$ ), providing the most unbiased samples of Compton-thick AGN in the local Universe. Owing to the limited imaging capabilities of these missions (coded-mask detectors), the flux limit probed is bright $\left(\sim 10^{-11} \mathrm{erg} \mathrm{cm}^{-2} \mathrm{~s}^{-1}\right)$, allowing only the detection of AGN at very low redshifts. These surveys did not detect large numbers of Compton-thick sources (Ajello et al. 2008, Tueller et al. 2008, Paltani et al. 2008,Winter et al. 2009, Burlon et al. 2011). The fraction of Compton-thick AGN in these surveys does not exceed a few percent of the total AGN population. However, Burlon et al. (2011) point out, that even these ultra-hard surveys are biased against the most heavily obscured $\left(>2 \times 10^{24} \mathrm{~cm}^{-2}\right)$ Compton-thick sources.

\subsection{Mid-IR selected $A G N$}

Brightman \& Nandra (2011) present XMM-Newton X-ray spectra for 126 sources from the $12 \mu \mathrm{m}$ sample of Rush et al. (1993). This sample contains 893 mid-IR selected local galaxies of which about 10\% are AGN. There are 12 Compton-thick AGN in this XMM-Newton sample according to the X-ray spectroscopic criteria, resulting in a fraction of Compton-thick AGN of about $20 \%$.

\subsection{Optically selected $A G N$}

Akylas \& Georgantopoulos (2009) present XMM-Newton spectral analysis of all 38 Seyfert galaxies from the Palomar spectroscopic sample of galaxies (Ho et al. 1997). These are found at distances of up to $67 \mathrm{Mpc}$ and cover the absorbed 2$10 \mathrm{keV}$ luminosity range $\mathrm{L}_{2-10} \sim 10^{38}-10^{43} \mathrm{erg} \mathrm{s}^{-1}$. Three of their sources are mildly Compton-thick with column densities just above $10^{24} \mathrm{~cm}^{-2}$ and high equivalent width FeK $\alpha$ lines $(>700 \mathrm{eV})$. When one considers only the 21 brightest sources with X-ray luminosity above $L_{X}=10^{41} \mathrm{erg} \mathrm{s}^{-1}$, which are contributing significantly to the X-ray background, the fraction of Compton-thick sources becomes $15 \%$. The Palomar spectroscopic survey finds AGN at very low redshifts. Attempts to detect Compton-thick AGN at higher redshifts, using the X-ray to [OIII] or [NeV] luminosity ratio have been performed by Vignali et al. (2004) and Gilli et al. (2010). 
Table 1. Compton-thick AGN fraction in the local Universe

\begin{tabular}{ccc}
\hline Survey & CT fraction & Ref \\
\hline IRAS-12 $\mu \mathrm{m}$ & 0.2 & Brightman \& Nandra 2011 \\
SWIFT & 0.04 & Burlon et al. 2011 \\
Palomar & 0.15 & Akylas \& Georgantopoulos 2009 \\
\hline
\end{tabular}

We summarize the comparison of the fraction of Compton-thick AGN, in the local Universe, from surveys in different wavelengths in Table 1.

\section{Deep X-Ray Surveys}

The deep surveys with Chandra and XMM provided the opportunity to search for Compton-thick AGN, mainly through X-ray spectroscopy, at faint fluxes and moderate redshifts $(z \sim 0.7-1)$ where the bulk of the $\mathrm{X}$-ray background is produced. The population synthesis models (Gilli et al. 2007) predict an upturn in the number of Compton-thick AGN at fluxes of $\sim 10^{-15} \mathrm{erg} \mathrm{cm}^{-2} \mathrm{~s}^{-1}(2-10 \mathrm{keV})$ about a factor of 20 brighter than the flux limit of the Chandra Deep Field South (CDF-S) 4Ms survey (Xue et al. 2011). Tozzi et al. (2006) and Georgantopoulos et al. (2007) first applied $\mathrm{X}$-ray spectroscopy in the $1 \mathrm{Ms}$ CDFS observations to identify reflection-dominated sources finding a couple of tens candidate Compton-thick sources. Georgantopoulos et al. (2009) repeated this exercise in the CDF-N. The above authors find 10 candidate Compton-thick sources down to a flux of $\mathrm{f}_{2-10} \sim 10^{-15} \mathrm{erg} \mathrm{cm}^{-2} \mathrm{~s}^{-1}$. The co-added spectrum of the six sources with available spectroscopic redshifts presents a FeK $\alpha$ line at $6.4 \mathrm{keV}$ rest-frame energy with an equivalent width of $\sim 1 \mathrm{keV}$ (Fig. 1 ) showing unambiguously that a large fraction of these are associated with Comptonthick sources. Comastri et al. (2011), using the 3Ms XMM-Newton observations in the CDF-S, re-examined the candidate Compton-thick sources found by Tozzi et al. (2006). They find at least two bona-fide Compton-thick sources, one at a redshift of $\mathrm{z}=1.53$ and another one at $\mathrm{z}=3.7$ (see also Norman et al. 2002), see Fig. 2. Feruglio et al. (2011) and Gilli et al. (2011) analysing Chandra data in the CDF-S find another two Compton-thick sources at high redshift. The one found by Gilli et al. (2007), at $\mathrm{z} \sim 5$, is the highest redshift AGN discovered so far.

\section{Mid-IR Surveys}

The mid-IR wavelengths have attracted much attention for providing an alternative way to detect heavily obscured systems. This is because the absorbed radiation by circumnuclear dust is re-emitted in the IR part of the spectrum (Soifer et al. 2008), rendering heavily obscured AGN copious mid-IR emitters. In such systems, the 2$10 \mathrm{keV}$ X-ray emission can be diminished by almost two orders of magnitude (Matt et al. 2004), while at the same time the isotropic mid-IR emission remains largely unattenuated. 


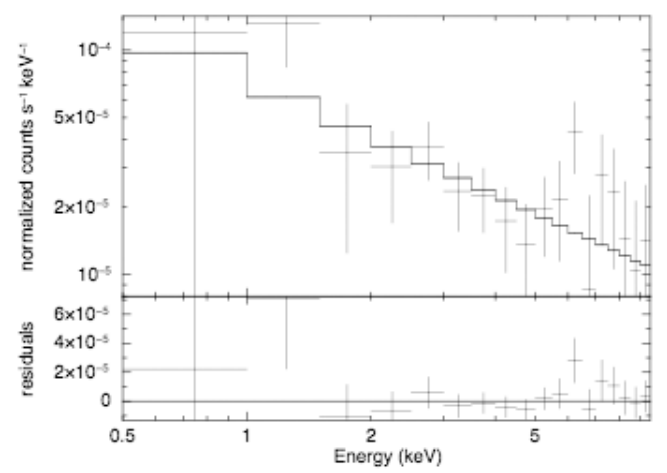

Fig. 1. The co-added X-ray spectrum and single power-law fit (as well as residuals) of the six sources in CDF-N with a reflected dominated spectrum and spectroscopic redshifts available (Georgantopoulos et al. 2009). A large equivalent-width $(\sim 1 \mathrm{keV}) \mathrm{FeK} \alpha$ line at a rest-frame energy of $6.4 \mathrm{keV}$ can be clearly seen.
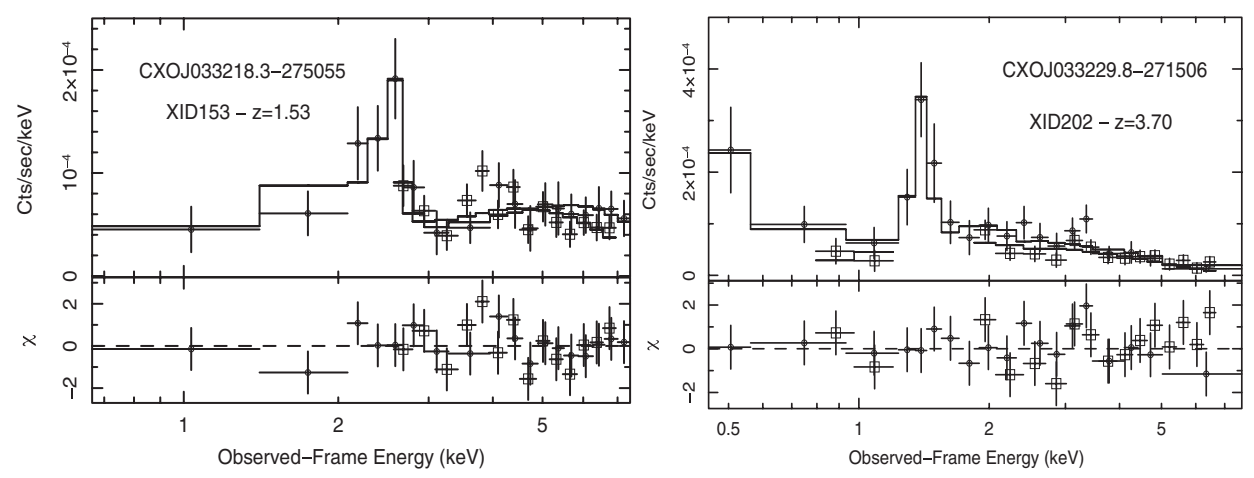

Fig. 2. The XMM-Newton spectra of the two high-redshjift Compton-thick sources presented in Comastri et al. (2011).

\subsection{X-ray to mid-IR luminosity ratio}

The presence of a low $\mathrm{L}_{\mathrm{X}} / \mathrm{L}_{6 \mu \mathrm{m}}$ luminosity ratio has been used as the main instrument for the selection of faint Compton-thick AGN which remain undetected in X-rays (e.g. Alexander et al., 2008; Goulding et al., 2011). This is because the $6 \mu \mathrm{m}$ luminosity is an excellent proxy of the AGN power, as it should be dominated by very hot dust heated by the AGN (e.g. Lutz et al., 2004). At these wavelengths, the contribution of the stellar light and colder dust heated by young stars should be minimal.

Georgantopoulos et al. (2011d) have assessed the efficiency of the X-ray ratio method, estimating the percentage of Compton-thick AGN among the low $\mathrm{L}_{\mathrm{X}} / \mathrm{L}_{6 \mu \mathrm{m}}$ sources. They used a sample of local AGN, the IRAS $12 \mu \mathrm{m}$ sample of Rush, Malkan 

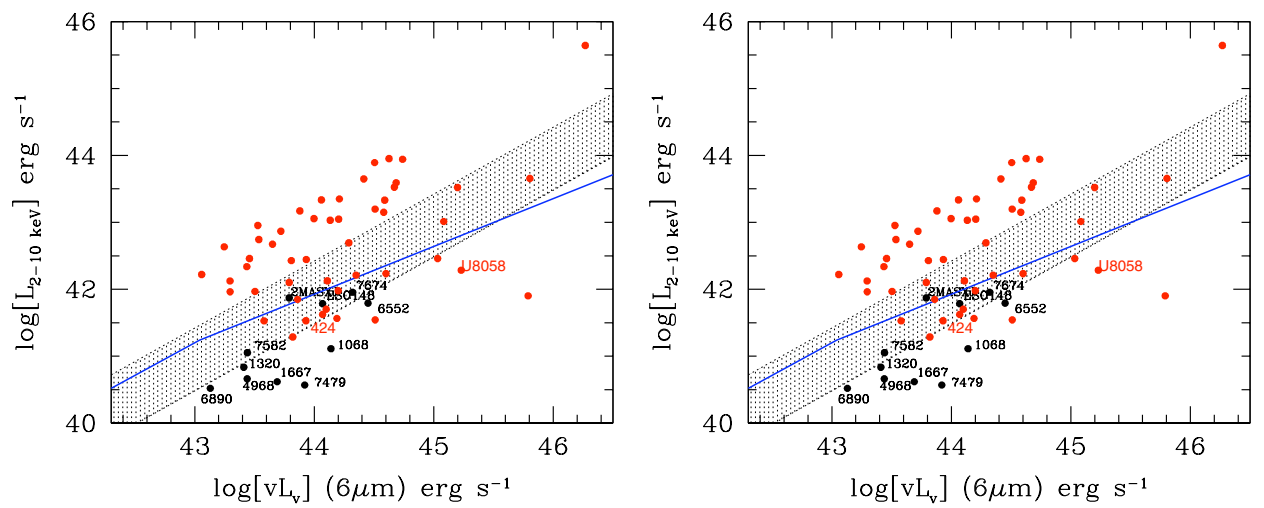

Fig. 3. Left panel: The X-ray $(2-10 \mathrm{keV})$ vs $6 \mu \mathrm{m}$ luminosity ratio for the IRAS $12 \mu \mathrm{m}$ sample of Brightman \& Nandra (2011). The black points denote the AGN which are unambiguously associated with Compton-thick sources on the basis of XMM-Newton spectroscopy. The blue line corresponds to the COSMOS average AGN $\mathrm{L}_{\mathrm{X}} / \mathrm{L}_{6} \mu \mathrm{m}$ luminosity ratio scaled down to $3 \%$ to account for the attenuation of X-rays in Compton-thick AGN. The hatched area corresponds to the local AGN X-ray to mid-IR luminosity relation (Lutz et al. 2004) and its associated $1 \sigma$ error reduced down to $3 \%$. Right panel: The filled circles denote the X-ray selected AGN in the CDFS with luminosity $\mathrm{L}_{\mathrm{X}}>10^{42} \mathrm{erg} \mathrm{s}^{-1}$. in the CDFS. The red squares correspond to the two bona-fide Compton-thick AGN in Comastri et al. (2011). Crosses denote the reflection-dominated Comptonthick AGN in Tozzi et al. (2006) while the open star corresponds to the Compton-thick AGN in Feruglio et al. (2011). For more details see Georgantopoulos et al. (2011d).

\& Spinoglio (1993). For this sample excellent quality X-ray spectroscopic observations are available (Brightman \& Nandra, 2011), and hence there is a priori knowledge on which objects are Compton-thick. The $\mathrm{L}_{\mathrm{X}} / \mathrm{L}_{6 \mu \mathrm{m}}$ diagram for the nearby sources is shown in Fig. 3. The X-ray luminosity is uncorrected for obscuration, while the $6 \mu \mathrm{m}$ luminosity includes both the torus and the star-formation component. Most (ten out of eleven) of the sources classified as Compton-thick on the basis of X-ray spectroscopy lie in the Compton-thick regime. However, many more sources (twelve) that are not Compton-thick, according to the XMM-Newton Xray spectroscopic diagnostics, would be classified as candidate Compton-thick AGN on the basis of the low $\mathrm{L}_{\mathrm{X}}-\mathrm{L}_{6 \mu \mathrm{m}}$ ratio.

In addition, the above authors select candidate Compton- thick AGN among the low $\mathrm{L}_{\mathrm{X}} / \mathrm{L}_{6 \mu \mathrm{m}}$ AGN in the CDF-S. In this field both Chandra (4Ms) and XMM $(3 \mathrm{Ms})$ data are available, rendering it the most well observed area in the X-ray sky. They cannot confirm that a large fraction of the low $\mathrm{L}_{\mathrm{X}} / \mathrm{L}_{6 \mu \mathrm{m}}$ CDF-S sources are Compton-thick on the basis of the X-ray spectroscopy. Nevertheless, these sources are highly obscured, having column densities above $10^{23} \mathrm{~cm}^{-2}$. Most importantly, they found that the two bona-fide Compton-thick sources from Comastri et al. (2011) do not appear to have a low $\mathrm{L}_{\mathrm{X}} / \mathrm{L}_{6 \mu} \mathrm{m}$ ratio. This suggests that the $\mathrm{X}$ ray to mid-IR luminosity cannot be used on its own to reliably classify sources as Compton-thick, casting doubt on the efficiency of this method. 


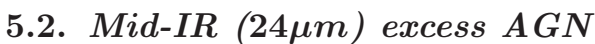

A number of authors have proposed that sources with mid-IR excess at $24 \mu \mathrm{m}$ are hosting heavily obscured and probably Compton-thick nuclei. Martinez-Sansigre et al. (2005) first argued that a population of bright $24 \mu \mathrm{m}$ AGN with no $3.6 \mu \mathrm{m}$ detection is as numerous as unobscured QSOs at high redshift $\mathrm{z}>2$. Houck et al. (2005) have detected a population of $24 \mu \mathrm{m}$ bright sources that are very faint in the optical R-band having $\mathrm{f}_{24 \mu \mathrm{m}} / \mathrm{f}_{\mathrm{R}}>1000$. These sources have been nicknamed DOGs (Dust Obscured Galaxies). The redshift of these sources is $\mathrm{z} \approx 2$ with a small scatter $\sigma_{z} \approx 0.5$ (Dey et al. 2008, Pope et al. 2008). Fiore et al. (2008), Georgantopoulos et al. (2008), Fiore et al. (2009), Treister et al. (2009b), Eckart et al. (2010), argue that the stacked X-ray signal of the $24-\mu \mathrm{m}$ infrared excess AGN are associated with heavily obscured AGN below the flux limit of the deepest Chandra surveys. These sources have a stacked signal with a spectrum of $\Gamma \sim 1$. Georgakakis et al. (2010), examine the X-ray spectra of a number of low redshift $(z \approx 1)$ analogs of DOGs, in the AEGIS and CDF-N surveys. They find that only three galaxies may show tentative evidence for Compton-thick obscuration. Finally, Georgantopoulos et al. (2011b) present an X-ray spectral analysis of all $24-\mu \mathrm{m}$ excess sources found in the Chandra deep fields (22 sources). They identify a number of at most 12 intrinsically flat $\Gamma<1$ sources which could be associated with Compton-thick sources. On the other hand, a large number of the remaining ten sources have steep X-ray spectra $(\Gamma>1.4)$. They conclude that the fraction of Compton-thick sources among DOGs cannot exceed roughly $50 \%$ among the $24 \mu \mathrm{m}$ excess sources.

\subsection{AGN with large optical depth Si $9.7 \mu \mathrm{m}$ absorption}

Observations with the IRS spectrograph onboard Spitzer have found many sources with very deep Si features at $9.7 \mu \mathrm{m}$, that have optical depths of $\tau>1$. Since it is believed that a few of these systems in the local Universe are associated with Compton-thick active galactic nuclei (e.g. NGC4945, Armus et al. 2007), it is interesting to investigate whether the presence of a strong Si absorption feature is a good indicator of a heavily obscured AGN. Georgantopoulos et al. (2011c) have compiled $\mathrm{X}$-ray spectroscopic observations, available in the literature, on the optically-thick $\left(\tau_{9.7 \mu \mathrm{m}}>1\right)$ sources from the $12 \mu \mathrm{m}$ IRAS Seyfert sample. They find that the majority of the high- $\tau$ optically confirmed Seyferts (six out of nine) in the $12 \mu \mathrm{m}$ sample are probably Compton-thick. Thus, there is direct evidence of a connection between mid-IR optically-thick galaxies and Compton-thick AGN, with the success rate being close to $70 \%$ in the local Universe. However, this technique cannot provide complete Compton-thick AGN samples, i.e., there are many Compton-thick AGN that do not display significant Si absorption, with the most notable example being NGC 1068.

In addition, Georgantopoulos et al. (2011c) have constructed a sample of candidate Compton-thick AGN at higher redshifts. This consists of seven high- $\tau$ Spitzer sources in the Great Observatories Origins Deep Survey (GOODS) and five in the 
Spitzer First-Look Survey. All these have been selected to have no PAH features $\left(\mathrm{EW}_{6.2 \mu \mathrm{m}}<0.3 \mu \mathrm{m}\right)$ to maximise the probability that they are bona-fide AGN. Six out of the seven GOODS sources have been detected in X-rays, while for the five FLS sources only X-ray flux upper limits are available. The high X-ray luminosities $\left(\mathrm{L}_{\mathrm{X}}>10^{42} \mathrm{erg} \mathrm{s}^{-1}\right)$ of the detected GOODS sources corroborates that these are AGN. For FLS, ancillary optical spectroscopy reveals hidden nuclei in two more sources. SED fitting can support the presence of an AGN in the vast majority of sources. Unfortunately, owing to the limited photon statistics, no useful constraints can be derived from X-ray spectroscopy. Therefore the efficiency of the high- $\tau$ method for finding Compton-thick AGN at high redshift remains uncertain.

\subsection{Compton-thick AGN in sub-mm galaxies}

The first extragalactic surveys at $850 \mu \mathrm{m}$ with the SCUBA detector on the JCMT telescope revealed a population of numerous luminous high redshift sub-millimeter galaxies or SMGs (see Blain et al. 2002, Maiolino 2008). Sub-mm surveys are very efficient in detecting distant galaxies because of the negative K-correction at submm wavelengths which counteracts the dimming of light with increasing distance. Chapman et al. (2005) have shown that the SMG population lies at high redshift with the median being $\mathrm{z}=2-3$, although the highest redshift SMGs have been found out to $z=4-5$ (Capak et al. 2008, Wardlow et al. 2010). Mid-IR spectroscopy reveals that the SMGs generate large fractions of their energy due to star-forming processes (Pope et al. 2008, Menendez-Delmestre 2009). Deep X-ray surveys allow the assessment of the AGN content of distant SMGs. Alexander et al. (2005a, 2005b) have found X-ray counterparts to a sample of 20 SMGs with radio counterparts from Chapman et al. (2005) in the Chandra Deep Field North (CDFN). Based on the number of X-ray detections, Alexander et al. (2005b) claim a high fraction $(75 \pm 19 \%)$ of AGN among the sub-mm galaxies which have radio counterparts. If one takes into account the radio undetected SMGs in the CDFN, making the conservative assumption that none of the radio undetected SMGs hosts AGN activity, then the AGN fraction in the SMG population becomes $>38_{-10}^{+12} \%$ (Alexander et al. 2005). Alexander et al. (2005b) argue that the vast majority of the radio-detected SMGs are highly obscured with column densities exceeding $10^{23} \mathrm{~cm}^{-2}$. The above results suggest that the intense star-formation goes hand-in-hand with supermassive black-hole growth. Laird et al. (2010) presented an analysis of 35 SMGs in the CDFN with sub-arcsec positions either from radio or Spitzer counterparts. Their $\mathrm{X}$-ray spectra and low X-ray luminosities reveal that a dominant AGN contribution is only required in only seven sources, or $20 \%$ of the SMG sample. Georgantopoulos et al. (2011b) studied the X-ray properties of the 126 sub-mm sources of the LABOCA survey in the area of the CDF-S and extended CDF-S. They find 14 sources detected in X-rays. The X-ray luminosities and spectra suggest that most of the sub-mm - X-rays associations (10/14) host an AGN while In four sources the X-ray emission could possibly originate from star-forming processes. Hence, the 
fraction of X-ray AGN among the LABOCA SMG sample in the area of the CDF-S is at most $26 \pm 9 \%$. Only six of the X-ray sources show large amounts of absorption, $\mathrm{N}_{\mathrm{H}}>10^{23} \mathrm{~cm}^{-2}$, but there is no unambiguous evidence that any of these is associated with a Compton-thick source. Finally, they argue that Compton-thick AGN do not even lie among the SMGs undetected at X-ray wavelengths. X-ray stacking analysis of the undetected SMGs reveals a signal with a relatively soft spectrum which is more suggestive of a SFR galaxy population.

\section{References}

1. Ajello, M., Rau, A., Greiner, J., et al. 2008, ApJ, 673, 96.

2. Akylas, A., Georgantopoulos, I., 2009, A\&A, 500, 999.

3. Alexander, D. M., Bauer, F. E., Brandt, W. N., et al., 2003, AJ, 126, 539.

4. Alexander, D.M., Smail, I., Bauer, F.E., Chapman, S. C., 2005, Nature, 434, 738.

5. Alexander, D.M., Bauer, F.E., Chapman, S.C. et al., 2005, ApJ, 632, 736.

6. Alexander, D. M., Chary, R. R., Pope, A., et al.. 2008, ApJ, 687, 835.

7. Alexander, D.M., Bauer, F.E., Brandt, W.N., et al.. 2011, astro-ph/1106.1443.

8. Ballantyne, D.R., Draper, A.R., Madsen, K.K., Rigby, J.R., Treister, E., 2011, ApJ, $736,56$.

9. Blain, A.W., Smail, I., Ivison, R.J., Kneib, J.P., Frayer, D.T., 2002, PhR, 369, 111.

10. Braito, V., Della Ceca, R., Piconcelli, E. et al.. 2004, A\&A, 420, 79.

11. Brandt W. N., Hasinger, G., 2005, ARA\&A, 43, 827.

12. Brightman, M., Nandra, K., 2011, MNRAS, 413, 1206.

13. Burlon, D., Ajello, M., Greiner, J., Comastri, A., Merloni, A., Gehrels, N., 2011, ApJ, 728,58 .

14. Capak, P., Carilli, C.L., Lee, N. et al.. 2008, 681, L53.

15. Chapman, S.C., Blain, A. W., Smail, I., Ivison, R. J., 2005, ApJ, 622, 772.

16. Churazov, E., Sunyaev, R., Revnivtsev, M., et al.. 2007, A\&A, 467, 529.

17. Comastri, A. 2004, ASSL, 308, 245.

18. Comastri, A., Iwasawa, K., Gilli, R., Vignali, C., Ranalli, P., Matt, G., Fiore, F., 2010, ApJ, 717, 787.

19. Comastri, A., Ranalli, P., Iwasawa, K., et al.., 2011, A\&A, 526, L9.

20. Dey, A., Soifer, B.T.,Desai, V. et al.., 2008, ApJ, 677, 943.

21. Daddi, E., Alexander, D. M., Dickinson, M., et al.., 2007, ApJ, 670, 173.

22. Dadina, M., 2007, A\&A, 461, 1209.

23. Donley, J. L., Rieke, G. H., Alexander, D. M., Egami, E., Pérez-González, P. G., 2010, ApJ, 719, 1393.

24. Eckart, M. E., McGreer, I. D., Stern, D., Harrison, F. A., Helfand, D. J., 2010, ApJ, 708,584 .

25. Feruglio, C., Daddi, E., Fiore, F., Alexander, D. M., Piconcelli, E., Malacaria, C., 2011, ApJ, 729, L4.

26. Fiore, F., Grazian, A., Santini, P., et al.., 2008, ApJ, 672, 94.

27. Fiore, F., Puccetti, S., Brusa, M., et al.., 2009, ApJ, 693, 447.

28. Frontera, F., Orlandini, M., Landi, R., 2007, ApJ, 666, 86.

29. Fukazawa, Y., Hiragi, K., Mizuno, M., et al.., 2011, ApJ, 727, 19.

30. Georgakakis, A., Hopkins, A.M., Sullivan, M., Afonso, J., Georgantopoulos, I., Mobasher, B., Cram, L.E., 2003, MNRAS, 345, 939.

31. Georgakakis, A., Rowan-Robinson, M., Nandra, K., Digby-North, J., Pérez-González, P. G., Barro, G., 2010, MNRAS, 406, 420. 
32. Georgantopoulos, I., Georgakakis, A., Akylas, A., 2007, A\&A, 466, 823.

33. Georgantopoulos, I., Georgakakis, A., Rowan-Robinson, M., Rovilos, E., 2008, A\&A, 484, 671 .

34. Georgantopoulos, I., Akylas, A., Georgakakis, A., Rowan-Robinson, M., 2009, A\&A, 507,747 .

35. Georgantopoulos, I., Rovilos, E., Comastri, A., 2011, A\&A, 526, 46.

36. Georgantopoulos, I., Rovilos, E., Xilouris, E.M., et al.., 2011b, A\&A, 526, 86.

37. Georgantopoulos, I., Dasyra, K., Rovilos, E., et al.., 2011c, A\&A, 531, 116.

38. Georgantopoulos, I., Rovilos, E., Akylas, A., et al.. 2011d, A\&A, 534, 23.

39. Gilli, R., Comastri, A., Hasinger, G., 2007, A\&A, 463, 79.

40. Gilli, R., Vignali, C., Mignoli, M., Iwasawa, K., Comastri, A., Zamorani, G., 2010, A\&A, 519, 92.

41. Gilli, R., Su, J., Norman, C., et al.., 2011, ApJ, 730, L28.

42. Goulding, A. D., Alexander, D. M., Mullaney, J. R., Gelbord, J. M., Hickox, R. C., Ward, M., Watson, M. G., 2011, MNRAS, 411, 1231.

43. Laird, E.S., Nandra, K., Pope, A., Scott, D., 2010, MNRAS, 401, 2673.

44. Luo, B., Bauer, F.E., Brandt, W.N., et al.. 2008, ApJS, 179, 19.

45. Lutz, D., Maiolino, R., Spoon, H. W. W., Moorwood, A. F. M., 2004, A\&A, 418, 465.

46. Marconi, A., Risaliti, G., Gilli, R., Hunt, L.K., Maiolino, R., Salvati, M., 2004, MNRAS, 351, 169.

47. Maiolino, R., 2008, NewA, 52, 339.

48. Matt, G., Bianchi, S., Guainazzi, M., Molendi, S., 2004, A\&A, 414, 155.

49. Menendez-Delmestre, K., Blain, A.W., Smail, I., 2009, ApJ, 699, 677.

50. Merloni, A., Heinz, S., 2008, MNRAS, 388, 1011.

51. Moretti, A., Pagani, C., Cusumano, G., et al.. 2009, A\&A, 493, 501.

52. Norman, C., Hasinger, G., Giacconi, R., et al.., 2002, ApJ, 571, 218.

53. Paltani, S., Walter, R., McHardy, I. M., Dwelly, T., Steiner, C., Courvoisier, T. J.-L., 2008, A\&A, 485, 707.

54. Pope, A., Chary, R.-R., Alexander, D.M., 2008, ApJ, 675, 1171.

55. Rush, B., Malkan, M. A., Spinoglio, L., 1993, ApJS, 89, 1.

56. Sazonov, S., Krivonos, R., Revnivtsev, M., Churazov, E., Sunyaev, R., 2008, A\&A, $482,517$.

57. Soifer, B. T., Helou, G., Werner, M., 2008, ARA\&A, 46, 201.

58. Soltan, A., 1982, MNRAS, 200, 115.

59. Tozzi, P., Gilli, R., Mainieri, V., et al.., 2006, A\&A, 451, 457.

60. Treister, E., Urry, C. M., Virani, S., 2009, ApJ, 696, 110.

61. Treister, E., Cardamone, C. N., Schawinski, K., et al.., 2009b, ApJ, 706, 535.

62. Tueller, J., Mushotzky, R.F., Barthelmy, S., Cannizzo, J.K., Gehrels, N., Markwardt, C.B., Skinner, G.K., Winter, L.M., 2008, ApJ, 681, 113.

63. Vignali, C., Alexander, D.M., Comastri, A., 2004, MNRAS, 354, 720.

64. Wardlow, J.L., Smail, I., Coppin, K.E.K. et al.., 2010, MNRAS.

65. Winter, L. M., Mushotzky, R. F., Reynolds, C. S., Tueller, J., 2009, ApJ, 690, 1322.

66. Wolf, C., Wisotzki, L., Borch, A., Dye, S., Kleinheinrich, M., Meisenheimer, K., 2003, A\&A, 408, 499.

67. Xue, Y.Q., Luo, B., Brandt, W.N., et al.., 2011, ApJ. 